\title{
Antineutrophil cytoplasmic antibodies in Chinese patients with tuberculosis
}

\author{
Gao Huan ${ }^{[1],[2], ~ G a o ~ Y a n g}{ }^{[2],[3]}$, Qu Xiao-yu ${ }^{[2]}, X u$ Jiancheng ${ }^{[4]}$ and Song Yan-qing ${ }^{[2]}$ \\ [1]. School of Pharmaceutical Sciences, Jilin University, Changchun, Jilin, China. \\ [2]. Department of Pharmacy, the First Hospital of Jilin University, Changchun, Jilin, China. \\ [3]. Department of Neurology, the First Hospital of Jilin University, Changchun, Jilin, China. \\ [4]. Department of Clinical Laboratory, the First Hospital of Jilin University, Changchun1, Jilin, China.
}

\begin{abstract}
Introduction: Based on reports, infection with Mycobacterium tuberculosis is believed to induce the development of antibodies that are considered to be biological indicators for the diagnosis of some other diseases. However, conflicting results have been published regarding the presence of antineutrophil cytoplasmic antibodies (ANCAs) in patients with tuberculosis. We aim to study the seroprevalence of ANCA in a population of Chinese patients with tuberculosis, which may lead to the misdiagnosis of vasculitic disorders. Methods: The study was conducted from January 2016 to May 2017 to evaluate the presence of ANCA in 103 Chinese patients using indirect immunofluorescent assay. An enzyme-linked immunosorbent assay was performed for anti-myeloperoxidase (MPO) and anti-proteinase 3 (PR3) detection. Results: Perinuclear ANCA (p-ANCA) was detected in $4.8 \%(5 / 103)$ of patients, whereas cytoplasmic ANCA (c-ANCA) was not detected; $1.9 \%(2 / 103)$ of patients with tuberculosis was positive for anti-MPO antibodies, and none had anti-PR3 antibodies. Both anti-MPO-positive patients were diagnosed with ANCA-associated vasculitides. Conclusions: ANCA positivity may be more related to vasculitis and immunological disorders than to a M. tuberculosis infection. Therefore, to improve diagnostic accuracy, patients with M. tuberculosis who are ANCA positive should be investigated for concurrent diseases, including the effects of drugs. Therefore, even in tuberculosis epidemic area, ANCA seropositivity, detected by ELISA, is still more suggestive of ANCA-associated vasculitides.
\end{abstract}

Keywords: Antineutrophil cytoplasmic antibodies. Tuberculosis. Proteinase 3. Myeloperoxidase. Seroprevalence.

\section{INTRODUCTION}

Mycobacterium tuberculosis infection is one of the major global public health threats because more than two billion people are estimated to be infected with tuberculosis ${ }^{1}$. Tuberculosis is associated with autoimmune diseases, including rheumatoid arthritis, multiple sclerosis, and vasculitis, possibly through molecular mimicry ${ }^{2}$. Antineutrophil cytoplasmic antibodies (ANCAs) are directed against cytoplasmic ANCA (c-ANCA) and perinuclear ANCA (p-ANCA) antigens and are associated with Wegener's granulomatosis, polyangiitis, microscopic polyangiitis, and other autoimmune disorders, which are also considered to be clinical markers for systemic vasculitic disorders. Some studies reported that ANCAs could be positive in infectious diseases, such as tuberculosis; however, conflicting results have also been reported ${ }^{3,4}$. In clinical practice, immunological diseases, such as granulomatosis with polyangiitis, can present with clinical features that overlap

Corresponding author: Dr. Song Yan-Qing

e-mail: 84679082@qq.com

Received 11 October 2017

Accepted 8 May 2018 with those of tuberculosis. Therefore, an ANCA test may help with differential diagnosis. This study aims to investigate the prevalence of serum ANCA positivity in Chinese patients with tuberculosis.

\section{METHODS}

This single-center retrospective study was conducted at the First Hospital of Jilin University in Northeast China between January 2016 and May 2017 in accordance with the Declaration of Helsinki, and the local Ethics Committee approved this study. All patients who participated in this study signed an informed consent form. Patients who were either untreated or were within 30 days of beginning their treatment for M. tuberculosis infection were included. Tuberculosis infection was diagnosed through sputum microbiology testing, clinical and radiological signs, and symptoms. The study participants underwent a detailed clinical history, including questions about musculoskeletal symptoms, duration of symptoms, and a history of medication use.

The presence of ANCA was determined via indirect immunofluorescence (IIF) using a commercially available kit (Cell Signaling Co.). Testing was performed according to the manufacturer's protocol. Serum samples were diluted 1:100 in 
phosphate buffered saline (PBS) and incubated in microplates coated with the specific antigen. Antigen-antibody binding was detected using an anti-human immunoglobulin conjugated with peroxidase and a 3,3',5,5'-tetramethylbenzidine (TMB) chromogenic substrate. After washing with PBS, the microplates were examined, using a fluorescence microscope. Serum samples were also tested for the presence of antibodies to proteinase 3 (PR3) and myeloperoxidase (MPO), using standardized kits (Fitzgerald Industries International). Therefore, $10 \mu \mathrm{L}$ of sample was combined with $990 \mu \mathrm{L}$ of sample buffer in a polystyrene tube and mixed well. Controls were ready to use and did not need to be diluted. PR3 and MPO were bound separately to the microwells. We added $100 \mu \mathrm{L}$ of each sample into the wells and incubated for 30 minutes at $20-28^{\circ} \mathrm{C}$. After discarding the supernatant, we washed it 3 times with $300 \mu \mathrm{L}$ wash solution; and then TMB substrate solution was added into each well. A stop solution was used to quench the reaction. Finally, horseradish peroxidase (HRP)-conjugated anti-human immunoglobulin $\mathrm{G}(\mathrm{IgG})$ was used to immunologically detect the bound patient antibodies forming a conjugate/antibody/antigen complex. The microplates were examined at $450 \mathrm{~nm}$ using a fluorescence microscope.

Statistical analysis of the data included the presentation of quantitative variables as means [ $95 \%$ confidence interval $(95 \%$ CI)] or median, and interquartile range; qualitative variables were expressed as percentages. Statistical Package for the Social Sciences (SPSS) software (SPSS Statistics for Windows, Version 17.0. Chicago: SPSS Inc.) was used for the statistical analysis.

\section{RESULTS}

The clinical characteristics of the patients with tuberculosis and confirmed infection with $M$. tuberculosis are summarized in Table 1. The demographic, clinical, and radiological characteristics of the 103 patients with M. tuberculosis were expressed in numbers and percentages, unless otherwise stated.

Of the 103 patients with M. tuberculosis included in this study, 54\% (56/103) were women with a mean age of 51 years. Thirty-four patients were in the early stages of their treatment for tuberculosis; treatment included rifampicin (RFP), isoniazid, and pyrazinamide. The median treatment duration was 8 days (interquartile range, 2-19 days).

Of the 103 individuals included in the study, six had tuberculosis involving more than two sites, including tuberculous peritonitis, pleuritis and pericarditis, maxillofacial tuberculosis, urologic tuberculosis, and spinal tuberculosis.
TABLE 1: Characteristics of 103 patients with tuberculosis.

\begin{tabular}{lc}
\hline Clinical characteristics & Number (\%) \\
\hline Women: men & $56: 47$ \\
Age in years $(95 \% \mathrm{Cl})$ & $51(30-72)$ \\
\hline Clinical presentation of TB & 98 \\
$\quad$ pulmonary & 5 \\
$\quad$ extrapulmonary & 6 \\
$\quad$ multiple (>2) sites & 45 \\
\hline Symptoms & \\
$\quad$ fever, weight loss, cough & 34 \\
\hline TB treatment & \\
rifampicin, isoniazid, and pyrazinamide & 84 \\
\hline Diagnosis of TB & $4+18-$ \\
$\quad$ abnormal chest X-ray & $38+21-$ \\
$\quad$ positive microbiology & \\
T-SPOT.TB & 6 \\
\hline Co-morbidities & 30 \\
$\quad$ diabetes & 19 \\
$\quad$ hypertension & \\
renal disease &
\end{tabular}

95\% Cl: 95\% confidence intervals; TB: tuberculosis; T-SPOT.TB: a T cell-based assay for the detection of infection with Mycobacterium tuberculosis.

A previous episode of tuberculosis, which had preceded the current infection by 2-31 years, was noted in 20 patients.

The serology testing for ANCA showed that p-ANCA was detected in 4.8\% (5/103) of patients, and c-ANCA was not observed in any patients (Table 2). MPO was detected in 2/103 patients, who were diagnosed with tuberculosis and ANCA associated vasculitides. There were 3/103 patients without anti-MPO or PR3 who had a low serum titer of p-ANCA (1:10), among which, two patients had diabetes complicated by infection, and one patient had anaphylactoid purpura [Immunoglobulin A/Henoch-Schönlein purpura (IgA/HSP)], which is a form of vasculitis.

\section{DISCUSSION}

Although the most common clinical presentation of Mycobacterium tuberculosis infection is pulmonary tuberculosis, with more than $10 \%$ of patients developing extra-pulmonary manifestations, which often delay the diagnosis and allow the chronic inflammation to progress. Patients with pulmonary tuberculosis present with typical clinical symptoms, including

TABLE 2: Antineutrophil cytoplasmic antibody test results for 103 patients with culture-positive tuberculosis.

\begin{tabular}{lccc}
\hline ELISA & \multicolumn{2}{c}{ Indirect immunofluorescence assay } \\
\cline { 2 - 4 } & negative & c-ANCA & p-ANCA \\
\hline Anti-PR3/anti-MPO negative & 98 & 0 & 3 \\
Anti-PR3 positive & 0 & 0 & 0 \\
Anti-MPO positive & 0 & 0 & 2 \\
\hline Total number & 98 & 0 & 5 \\
\hline
\end{tabular}

ELISA: enzyme-linked immunosorbent assay; ANCA: antineutrophil cytoplasmic antibody; PR3: proteinase 3; MPO: myeloperoxidase; c-ANCA: cytoplasmic ANCA; p-ANCA: perinuclear ANCA. 
fever, cough, and hemoptysis, and with typical diagnostic findings that include nodular and cavitating lesions on chest imaging; chronic inflammation with necrotizing granuloma formation is histologically observed on lung biopsy. However, patients with autoimmune disorders, including systemic lupus erythematosus, and patients with systemic vasculitis, including Wegener's granulomatosis, share similar clinical and histological features with tuberculosis ${ }^{5-8}$.

The detection of serum antibodies against MPO can be associated with microscopic periarteritis (MPA) and necrotic and crescentic glomerulonephritis (NCGN). The anti-MPO antibody titer is associated with the disease activity and can be used for early diagnosis, prediction of disease recurrence, and guidance of patient response to clinical treatment. Anti-MPO antibody positivity is highly suggestive of MPA and NCGN. The presence of serum PR3 antibodies are indicative of Wegener's granulomatosis, which can be considered as a primary vasculitis.

Previously published studies showed that patients with tuberculosis may have serum ANCA, as well as anti-MPO, and anti-PR3. Clinical studies on the presence of ANCA in patients with tuberculosis have been controversial. As shown in Table 3, p-ANCA was the predominant pattern in four studies that included ANCA-positive patients. However, FlorezSuarez et al. reported that $80 \%$ of ANCA-positive patients had c-ANCA, and $44.4 \%$ of patients with tuberculosis showed ANCA positivity, and that $90 \%$ of ANCA-positive patients had anti-PR3 and anti-MPO 9 . The proportion of serum-positive tuberculosis patients has been shown to decrease during tuberculosis therapy ${ }^{10}$. In another study, p-ANCA was detected in $25 \%$ of Iranian patients with tuberculosis, and c-ANCA was detected in $3.1 \%$; ELISA results showed that $75 \%$ of cases had anti-MPO, and $12.5 \%$ had anti-PR3, indicating a high ANCA seropositivity rate in tuberculosis ${ }^{3}$. However, Teixeira et al. showed that tuberculosis was associated with low ANCA (10\%) seroprevalence, including $4 \%$ of c-ANCA and $6 \%$ of p-ANCA, but only one IIF-negative specimen was anti-PR3 positive ${ }^{11}$.

The clinical distinction between tuberculosis and Wegener's granulomatosis can be difficult at disease onset. According to some studies, ANCA testing has an important role in the differential diagnosis. However, there have been conflicting results regarding the presence of ANCA in tuberculosis. Although reasons for the conflicting findings from these studies are not clear, it is likely that geographical factors and ethnic factors may have accounted for the differences. Additionally, drugs, such as hydralazine and propylthiouracil are associated with ANCA. Ori Elkayam ${ }^{12}$ have shown that most patients with serum anti-MPO normalized following the treatment, whereas anti-PR3 usually increased in some treated patients, which is probably due to drug-related autoimmune phenomena ${ }^{2,12}$. Esquivel-Valerio et al. found that in 68 cases, tuberculosis patients with ANCA positivity detected by an indirect immunofluorescent assay (IIF) was increased from $4.4 \%$ (one c-ANCA and two p-ANCA) to $28.8 \%$ ( 3 c-ANCA and 12 p-ANCA) after treatment, while anti-PR3 and anti-MPO were negative in all serum samples from patients with $\mathrm{TB}^{10}$. A published case report has shown that following treatment with RFP and ethambutol, serum anti-MPO and ANCA titers of the patient were high and a drug-induced nephritis was found ${ }^{13}$. Another case report showed that the serum

TABLE 3: Clinical studies on ANCA, anti-MPO, and anti-PR3 in patients with tuberculosis.

\begin{tabular}{|c|c|c|c|c|c|c|}
\hline Author & $\begin{array}{l}\text { Patients } \\
\text { with TB }\end{array}$ & $\begin{array}{c}\text { ANCA-positive } \\
\text { rate }(\%)\end{array}$ & p-ANCA $(\%)^{*}$ & C-ANCA $(\%)^{*}$ & Anti-MPO $(\%)^{*}$ & Anti-PR3 $(\%)^{*}$ \\
\hline Sherkat, et al ${ }^{3}$ & 32 & 28.1 & 89.0 & 11.0 & 75.0 & 12.5 \\
\hline Lima, et $\mathrm{al}^{4}$ & 50 & 0.0 & 0.0 & 0.0 & 0.0 & 0.0 \\
\hline Esquivel, et al ${ }^{10}$ & 68 & 4.4 & 66.7 & 33.3 & 0.0 & 0.0 \\
\hline Teixeira, et al ${ }^{11}$ & 67 & 10.0 & 60.0 & 40.0 & 0.0 & 14.3 \\
\hline Ghosh, et al ${ }^{16}$ & 70 & 30.0 & 52.4 & 47.6 & 47.6 & -- \\
\hline
\end{tabular}

ANCA: antineutrophil cytoplasmic antibody; anti-MPO: anti-myeloperoxidase; anti-PR3: anti-proteinase 3; MPO: myeloperoxidase; PR3: proteinase 3; TB: tuberculosis; p-ANCA: perinuclear ANCA; c-ANCA: cytoplasmic ANCA. ${ }^{*}$ The percentage of p-ANCA, c-ANCA, anti-MPO and anti-PR3 = positive number $/$ ANCA positive number $\times 100 \%$. 
anti-PR3 was positive after anti-tuberculosis treatment with RFP, which indicated a strong correlation between RFP and $\mathrm{ANCA}^{14}$. We consider that the presence of ANCA might be due to drugrelated autoimmune phenomena and, in some cases, might be affected by anti- tuberculosis treatment to a certain degree, with an increase in antibody titers to MPO and PR $3^{12}$. Our study, which included 103 patients with a confirmed diagnosis of TB on chest $\mathrm{X}$-ray and sputum microbiology, showed that the seroprevalence of ANCA was 4.9\% (5/103), which was low. The patients with positive ANCA, especially with anti-MPO, tended to have other additional diseases, which included ANCA-associated vasculitis in two patients. In our study, the positive rate of serum p-ANCA was $40 \%$ in the ANCA positive patients. Although anti-MPO positivity was found, no anti-PR3 was detected. Based on these findings, we conclude that anti-PR3/c-ANCA is a rare occurrence in tuberculosis, and that autoimmune disease or vasculitis should be considered in the differential diagnosis, and that other diseases should be excluded, especially in tuberculosis endemic areas.

Compared with previous studies ${ }^{1,3,4,10,11,15-17}$, this study recruited patients with many types of tuberculosis, including spinal tuberculosis, peritoneal tuberculosis, and tuberculous meningitis. Based on the findings of this study, patients who were ANCA-positive (especially for MPO or PR3) were more likely to have other ANCA-related disorders. Therefore, concurrent diseases, including the effects of drugs should initially be considered when ANCA positivity is found, even in countries with a high prevalence of tuberculosis.

This study had several limitations. It had a small study population and was performed in China. Therefore, the findings of this study in a Chinese population with tuberculosis may not be applicable to other geographical areas or other ethnic groups, although it indicates a low seroprevalence of ANCA in China. However, the study findings, when considered together with previously published studies, support that ANCA positivity without microbiological confirmation of tuberculosis is more likely to be due to systemic vasculitis.

\section{Ethical considerations}

All procedures performed in studies involving human participants were in accordance with the ethical standards of the institutional and/or national research committee and with the 1964 Helsinki Declaration and its later amendments, or comparable ethical standards. Formal consent was not required for this type of study.

\section{Conflict of interest}

The authors declare that there is no conflict of interest.

\section{Financial support}

National Natural Science Foundation of China: 81503168. Graduate Innovation Fund of Jilin University: 2017049.

\section{REFERENCES}

1. Pradhan VD, Badakere SS, Ghosh K, Pawar AR. Spectrum of anti-neutrophil cytoplasmic antibodies in patients with pulmonary tuberculosis overlaps with that of Wegener's granulomatosis. Indian J Med Sci. 2004;58(7):283-8.

2. Siddiqui MA, Khan IA. Isoniazid-induced lupus erythematosus presenting with cardiac tamponade. Am J Ther. 2002;9(2):163-5.

3. Sherkat R, Mostafavizadeh K, Zeydabadi L, Shoaei P, Rostami S. Antineutrophil cytoplasmic antibodies in patients with pulmonary tuberculosis. Iran J Immunol. 2011;8(1):52-7.

4. Lima I, Oliveira RC, Cabral MS, Atta A, Marchi S, Reis E, et al. Anti-PR3 and anti-MPO antibodies are not present in sera of patients with pulmonary tuberculosis. Rheumatol Int. 2014;34(9):1231-4.

5. Pradhan VD, Badakere SS, Iyer YS, Kumar R, Almeida AF. A study of anti-neutrophil cytoplasm antibodies in systemic vasculitis and other related disorders. J Postgrad Med. 2003;49(1):5-9.

6. Pradhan V, Badakere SS, Shankar Kumar U. Increased incidence of cytoplasmic ANCA (cANCA) and other autoantibodies in leprosy patients from western India. Lepr Rev. 2004;75(1):50-6.

7. Adebajo AO, Charles P, Maini RN, Hazleman BL. Autoantibodies in malaria, tuberculosis and hepatitis B in a west African population. Clin Exp Immunol. 1993;92(1):73-6.

8. Yahya TM, Benedict S, Shalabi A, Bayoumi R. Anti-neutrophil cytoplasmic antibody (ANCA) in malaria is directed against cathepsin G. Clin Exp Immunol. 1997;110(1):41-4.

9. Flores-Suarez LF, Cabiedes J, Villa AR, van der Woude FJ, AlcocerVarela J. Prevalence of antineutrophil cytoplasmic autoantibodies in patients with tuberculosis. Rheumatology (Oxford). 2003;42(2):223-9.

10. Esquivel-Valerio JA, Flores-Suárez LF, Rodríguez-Amado J, GarzaElizondo MA, Rendón A, Salinas-Carmona MC. Antineutrophil cytoplasm autoantibodies in patients with tuberculosis are directed against bactericidal/permeability increasing protein and are detected after treatment initiation. Clin Exp Rheumatol. 2010; 28 (1 Suppl 57):35-9.

11. Teixeira L, Mahr A, Jaureguy F, Noel LH, Nunes H, Lefort A, et al. Low seroprevalence and poor specificity of antineutrophil cytoplasmic antibodies in tuberculosis. Rheumatology (Oxford). 2005;44(2):247-50.

12. Elkayam O, Bendayan D, Segal R, Shapira Y, Gilburd B, Reuter $\mathrm{S}$, et al. The effect of anti-tuberculosis treatment on levels of antiphospholipid and anti-neutrophil cytoplasmatic antibodies in patients with active tuberculosis. Rheumatol Int. 2013;33(4):949-53.

13. Tanaka H, Oshiro Y, Kawanaka N, Kawasaki F, Okimotoi N. A case of MPO-ANCA-related nephritis caused by an anti-tuberculosis drug. Nihon Jinzo Gakkai shi. 2013;55(2):172-6.

14. Ji G, Zeng X, Sandford AJ, He JQ. Rifampicin-induced antineutrophil cytoplasmic antibody-positive vasculitis: a case report and review of the literature. Int J Clin Pharmacol Ther. 2016;54(10):804-7.

15. Flores-Suarez LF. Prevalence of antineutrophil cytoplasmic autoantibodies in patients with tuberculosis. Rheumatology (Oxford). 2003;42(2):223-9.

16. Ghosh K, Pradhan V, Ghosh K. Background noise of infection for using ANCA as a diagnostic tool for vasculitis in tropical and developing countries. Parasitol Res. 2008;102(5):1093-5.

17. Malacarne J, Rios DP, Silva CM, Braga JU, Camacho LA, Basta PC. Prevalence and factors associated with latent tuberculosis infection in an indigenous population in the Brazilian Amazon. Rev Soc Bras Med Trop. 2016;49(4):456-64. 